\title{
A pancake-shaped nano-aggregate for focusing surface plasmons
}

\author{
Shao Ying Huang, ${ }^{1,2,3}$ Weng Cho Chew, ${ }^{1,4}$ Yang G. Liu, ${ }^{5}$ Bae-lan Wu, ${ }^{3}$ and H. W. Choi ${ }^{1, a)}$ \\ ${ }^{1}$ Department of Electrical and Electronic Engineering, The University of Hong Kong, Hong Kong, China \\ ${ }^{2}$ Singapore University of Technology and Design, Singapore \\ ${ }^{3}$ Research Laboratory of Electronics, Massachusetts Institute of Technology, Cambridge, Massachusetts \\ 02139, USA \\ ${ }^{4}$ University of Illinois at Urbana-Champaign, Champaign, Illinois 61801, USA \\ ${ }^{5}$ Institute of Applied Physics and Computational Mathematics, Beijing, China
}

(Received 28 October 2011; accepted 23 December 2011; published online 8 February 2012)

\begin{abstract}
We proposed a pancake-shaped nano-aggregate that highly focuses surface plasmons. The structure is a superposition of bowtie-shaped dimers, where surface plasmons are excited, resonated with the structure, and coupled. Surface integral equation method (Poggio-Miller-Chang-Harrington-Wu-Tsai method) is used to predict the performance of the proposed structure. It is a method which can accurately calculate the near-fields of nanoparticles. Based on the numerical prediction, the proposed structure shows an electric field (E-field) enhancement of more than 400 times, which is equivalent to a Raman enhancement factor of more than $2.5 e 10$ times. It is promising for single molecule detections using surface-enhanced Raman scattering. The physics of the proposed structure are revealed. It is useful to design nanostructures for high E-field enhancement. (C) 2012 American Institute of Physics. [doi:10.1063/1.3681377]
\end{abstract}

\section{INTRODUCTION}

Surface-enhanced Raman scattering (SERS) has been a topic of strong scientific interest since 1997, when it was reported that single molecules absorbed on single nanoparticles can be detected using a methodology based on SERS. ${ }^{1,2}$ That was more than 20 years after the first discovery of SERS in 1974. The ability to detect single molecules makes SERS important in many fields, such as chemistry, biology, medicine, pharmacology, and environmental science. High enhancement factors are important in single molecule detections. The SERS enhancement factor in the single molecule detection experiment in Ref. 1 has been reported to be as high as $10^{14}-10^{15}$. Numerous studies are dedicated to model and analyze the giant enhancement factor in the experiment. ${ }^{3,4}$ Experimentally, progress in nanofabrication techniques has been reported, ${ }^{5,6}$ which enables the practical realizations of SERS-active substrates.

In SERS, the electric fields (E-fields) at the surface of nanoparticles are enhanced by a large factor mainly due to the excitation of surface plasmons at the incident frequency, $\omega_{i}$. With the excitation of surface plasmons near nanoparticles, the incident E-field is enhanced and induces dipoles in the molecules adsorbed at the surface of the particle. The induced dipoles are oscillating and produce Raman-scattered fields at a shifted frequency, $\omega_{S},{ }^{1,7}$ which is called Ramanshifted or Stokes-shifted frequency. Being similar to what happens to the incident fields, the Raman-scattered field excites the surface plasmons near the nanoparticles and is enhanced again. In other words, SERS is the process that the incident field is enhanced by the nanoparticle, shifted and scattered by the molecule, and enhanced for the second time

a)Electronic address: hwchoi@hku.hk. by the nanoparticle. More simply put, the incident field is enhanced twice with the assistance of the adsorbed molecules at the surface of the particles, and its frequency is shifted due to the nature of Raman scattering (inelastic scattering). Due to the localization of surface plasmons, the enhanced E-fields are localized near the nanoparticles.

With the mechanisms above, the SERS enhancement factor is proportional to $\left|\mathbf{E}\left(\omega_{i}\right) / \mathbf{E}_{i}\left(\omega_{i}\right)\right|^{2}\left|\mathbf{E}\left(\omega_{S}\right) / \mathbf{E}_{i}\left(\omega_{i}\right)\right|^{2}$, where $\mathbf{E}\left(\omega_{i}\right)$ and $\mathbf{E}\left(\omega_{S}\right)$ are the average E-fields on the particle surface at $\omega_{i}$ and $\omega_{S}$, respectively. ${ }^{8}$ When the enhancements at the two frequencies are close to each other, the enhancement factor is approximately equal to $\left|\mathbf{E}\left(\omega_{i}\right) / \mathbf{E}_{i}\left(\omega_{i}\right)\right|^{4}$.

In SERS experiments, the amplification of E-fields, $\mathbf{E}\left(\omega_{i}\right) / \mathbf{E}_{i}\left(\omega_{i}\right)$, is directly related to the SERS enhancement factor, $\left|\mathbf{E}\left(\omega_{i}\right) / \mathbf{E}_{i}\left(\omega_{i}\right)\right|^{2}\left|\mathbf{E}\left(\omega_{S}\right) / \mathbf{E}_{i}\left(\omega_{i}\right)\right|^{2}$. Numerous studies on the enhancement of E-fields for Raman-active substrates have been reported, where nanostructures with high enhancement factors have been proposed and demonstrated using experimental or/and numerical data. Due to the high enhancement of E-fields, the nanoparticles that excite surface plasmons are sometimes called optical antennas. ${ }^{9,10}$ The enhancement of E-fields is dependent on a number of factors, for example, the shape/geometry of the particles and their physical size with respect to the incident wavelength. Nanoparticles of different shapes/geometries, such as nanowells ${ }^{11}$ and nanocrescents, ${ }^{4}$ were fabricated, and SERS enhancement was demonstrated. The arrangement of particles, such as the number of particles, the periodicity, and the space between particles, is another factor that affects the SERS enhancement factor. A dimer, which has two particles in close proximity, shows a much higher enhancement factor than an isolated particle. $^{3}$ An array of particles obtains an extra factor of $10^{2}$ due to long-range photonic interactions caused by the periodicity. ${ }^{7}$ The space between two particles plays an important role in 
the enhancement. ${ }^{12}$ The enhancement factors increase as the distance between the two particles decreases. Besides the particles and their arrangements, the substrate that is used to support the particles shows effects on the enhancement of E-fields. ${ }^{13,14}$

Due to the dominant effect of the electromagnetic enhancement mechanism in SERS, electromagnetic theories analyzing the E-fields around the nanoparticles are useful in providing basic guidance to obtain a high Raman enhancement factor. For an isolated spherical nanoparticle, Mie theory provides an analytical solution. Numerically, finitedifference time-domain (FDTD) methods and finite element method (FEM) are straight-forward ones based on differential equation formulations, but they need to discretize both the objects and the surrounding space for solving the problem. Integral equation methods only need to discretize the objects, and the boundary conditions are rigorously included in the calculations. Volume integral equation formulation, including the discrete dipole approximation (DDA), results in full matrices with high cost in memory and computational time. When the object is homogeneous or is inhomogeneous with piecewise constant projection, surface integral equation (SIE) formulation is a good option. It is an efficient method, providing high accuracy in the near field and for the structures with singularities (sharp tips in the geometry of the nanoparticles).

In this paper, we propose an aggregate consisting of sectors of a circular cylinder. The performance of the proposed structure is predicted using method of moments (MoM). The proposed structure is predicted to have high enhancement of E-fields. The physics of the high performance are revealed. Variants of the proposed structure and their performance are discussed.

\section{DESIGN OF THE AGGREGATE}

Figure 1(a) shows the geometry of this proposed aggregate. As shown in Fig. 1(a), it consists of sectors of a circular cylinder. The central angle is denoted using $\theta$, the radius of the sector is $r$, and the thickness of the cylinder in the $z$-direction is $t$. All the cylinders are arranged together to form an aggregate. The insert in Fig. 1(a) shows the crosssectional view of the center of this aggregate. It is zoomed in and shown in Fig. 1(b). As shown in Fig. 1(b), the tip of a circle sector is truncated $a$ away from the tip. The distance between the two circle sectors along the same direction is $d$. The distance between the adjacent circle sectors is $g$. The adjacent circle sectors do not touch each other and $g \neq 0$.

The design starts from a bowtie-shaped dimer, as shown in Fig. 2(a). The design of the aggregate involves the dimension of the circular sectors $(r)$, the gap size $(d)$, and the super position of the dimers.

\section{A. Cross-sectional dimension of the nanoparticle}

The bowtie-shaped dimer shown in Fig. 2(a) was used to confine coherent mid-infrared radiation well below the diffraction limit in Ref. 9. In Ref. 9, numerical results show the high E-field enhancement in the gap of the dimer. In this section, more physics are revealed to show the advantages of a bowtie-shaped dimer.

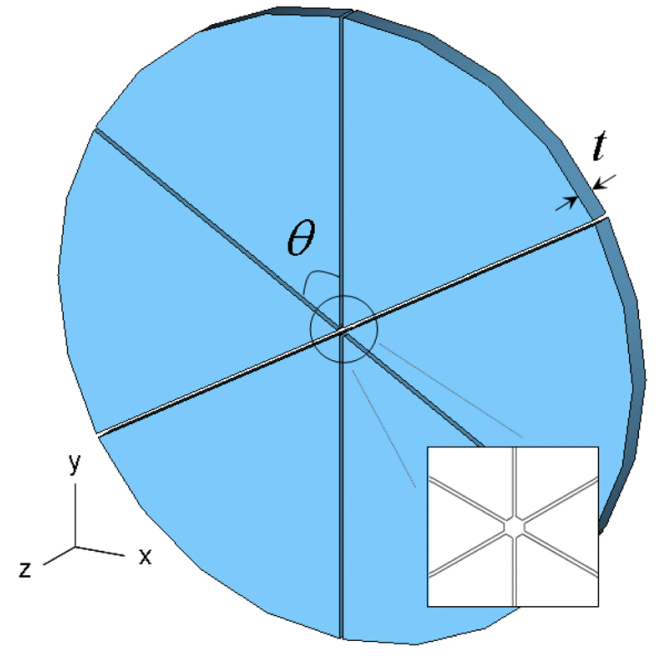

(a) $3 \mathrm{D}$ view

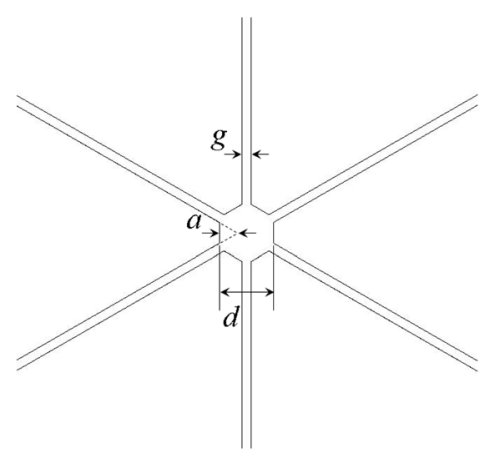

(b) Cross-sectional view

FIG. 1. (Color online) The proposed aggregate consisting of six cylinders with equilateral triangular cross-sections.

The bowtie-shaped dimer can be modeled using a wire dipole antenna excited by a current source, as shown in Fig. 2(b). In Fig. 2(b), the wires are aligned to the $x$-axis. For a wire antenna, the total length is set to be around halfwavelength to maximize the fields. ${ }^{15}$ For the bowtie-shaped dimer shown in Fig. 2(a), when the incident wave has the E-field aligned to the $x$-axis, the maximum dimension of the dimer in this direction $(2 r+d)$ is set to be half of the guided wavelength of the electromagnetic waves propagating in the particle along the same direction, $\lambda_{g} / 2{ }^{16}$ As $d \ll 2 r$, the optimal $r$ is set to be slightly less than a quarter of the guided wavelength. The guided wavelength of the waves in the particle is obtained based on the propagation of surface plasmons in the metal with a flat surface.

When a transverse magnetic (TM) plane wave impinges on a metal-dielectric surface, surface plasmons (surface plasmonic waves) are excited and propagate along the interface. ${ }^{17}$ Figure 3 shows the cross-sectional view of the dimer to illustrate the excitation of surface plasmons. The incident wave propagates in the $-z$-direction, and the E-field is along the $x$-direction. The incident wave excites surface plasmons at the metal-dielectric interface. The details of excitation are discussed in Ref. 17. It could be achieved by periodic patterns on the surface, which compensates the phase difference between lights and surface plasmons. When surface 


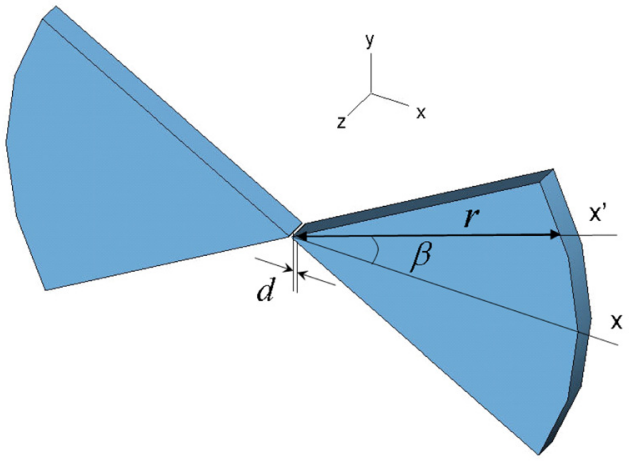

(a) a bowtie-shaped dimer

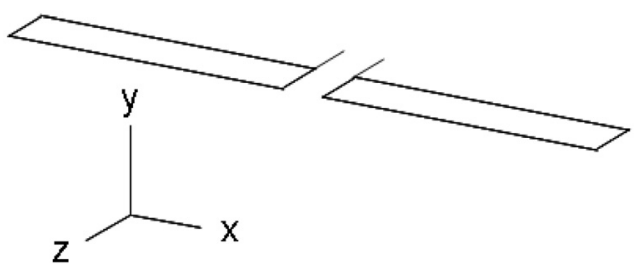

(b) a wire antenna

FIG. 2. (Color online) The geometry of a bowtie-shaped dimer and a wire antenna.

plasmons are excited, they are propagating along the $x$-direction and decay rapidly in the $+z$ - and $-z$-direction. They are surface waves. The value of the $k$-vector in the $x$-direction is expressed as follows: ${ }^{17}$

$$
k_{x}=\frac{\omega}{c}\left(\frac{\epsilon_{1} \epsilon_{2}}{\epsilon_{1}+\epsilon_{2}}\right)^{\frac{1}{2}},
$$

where $\omega$ is the angular frequency of the incident wave, $c$ is the speed of light in the vacuum, and $\epsilon_{1}$ and $\epsilon_{2}$ are the dielectric constants of the metal and the dielectric, respectively. Thus, in the $x$-direction,

$$
\lambda_{g}=\frac{2 \pi}{k_{x}}
$$

Surface plasmons are excited along the interface propagating at $\lambda_{g}$. When the surface is carved for the fabrication of nanoparticles, resonance can be obtained along the direction of propagation if the dimension of the particle is set properly. For a bowtie-shaped dimer, the radius of the sector, $r$, is set slightly less than $\lambda / 4$ to excite the structure resonance. With $\lambda_{g}$ expressed in Eq. (2), $r$ in this direction is obtainable.

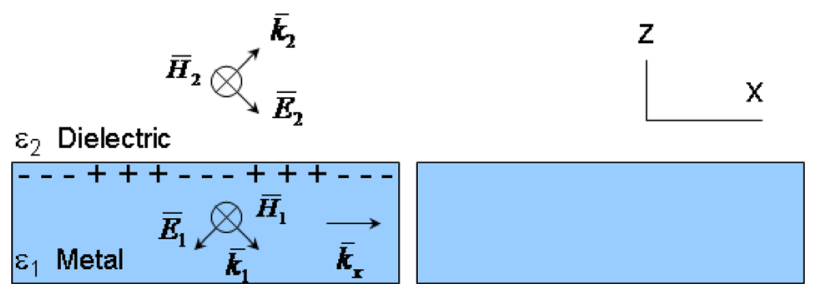

FIG. 3. (Color online) Two-dimensional view of a bowtie-shaped dimer to illustrate the excitation of surface plasmons.
When the direction is at an angle $\beta$ from the $x$-direction, as shown in Fig. 2(a), the optimal $r$ remains the same. The explanations are as follows: At angle $\beta$, we denote the direction as $x^{\prime}$-direction. In the $x^{\prime}$-direction, surface plasmons are excited in the dimer and resonate at the radius $r$ calculated from Eqs. (1) and (2). When the incident wave $\left(\mathbf{k}_{i n c}=-k_{z} \hat{z}\right.$, $\mathbf{E}_{i n c}=E_{x} \hat{x}$ ) illuminates the dimer, the incident waves can be decomposed into TM and transverse electric (TE) waves with respect to the new axis, $x^{\prime}$-axis. The new TM waves excite surface plasmons along the $x^{\prime}$-axis. In the new direction, the $\mathrm{k}$-vector of the surface plasmonic wave is still governed by Eq. (1). As can be seen in Eq. (1), it is determined by the two materials at the interface as well as the working frequency. Therefore, the k-vector of the surface plasmonic wave in a dimer is not affected by the change of $\beta$, neither is the optimal radius of the sectors of the dimer, $r$. Therefore, the optimal $r$ is fixed at different $\beta \mathrm{s}$, leading to two circular sectors pointing to each other, as shown in Fig. 2(a). For a dimer with two cylindrical components, it can be concluded that the E-field is amplified maximally when the dimer is constructed by two sectors of a circular cylinder with an optimized radius based on Eq. (2), meaning $r(\beta)$ is fixed to the value calculated based on Eq. (2).

\section{B. Superposition of dimers}

Figure 4 shows a dimer that is rotated at an angle $\phi$ anticlockwise from the $x$-axis. There are surface plasmons excited in the rotated dimer with the same incident waves $\left(\mathbf{k}_{i n c}=-k_{z} \hat{z}, \mathbf{E}_{i n c}=E_{x} \hat{x}\right)$. The explanations are similar to those shown in Sec. II B. The incident waves can be decomposed into TE and TM waves with respect to the new axis, $x^{\prime}$-axis, which is the axis passing through the center of the rotated dimer. The new TM waves excite surface plasmons along the $x^{\prime}$-axis. In the rotated dimer, the k-vector of the surface plasmonic wave is still governed by Eq. (1). As can be seen in Eq. (1), it is determined by the two materials at the interface as well as the working frequency. Therefore, the $\mathrm{k}$-vector of the surface plasmonic wave in a dimer is not affected by the rotation, neither is the optimal radius of the sectors of the dimer $(r)$. Therefore, for the rotated dimers,

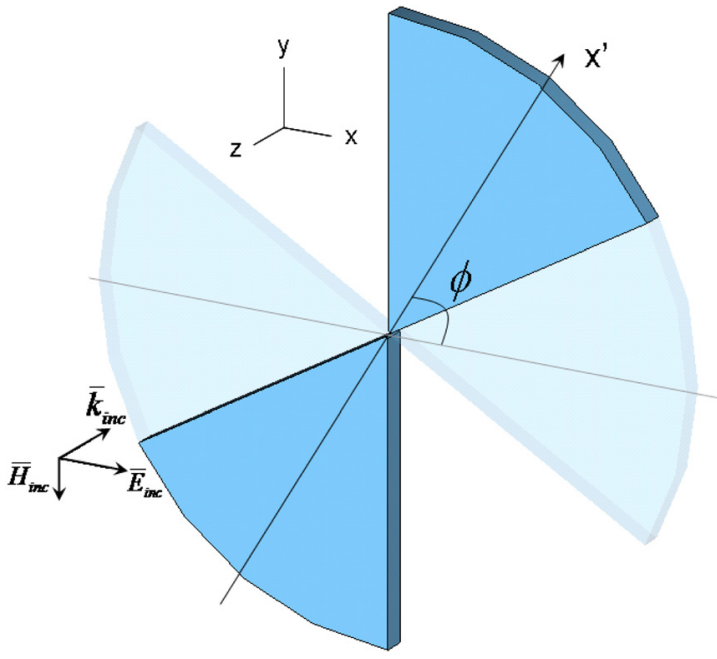

FIG. 4. (Color online) A rotated dimer. 


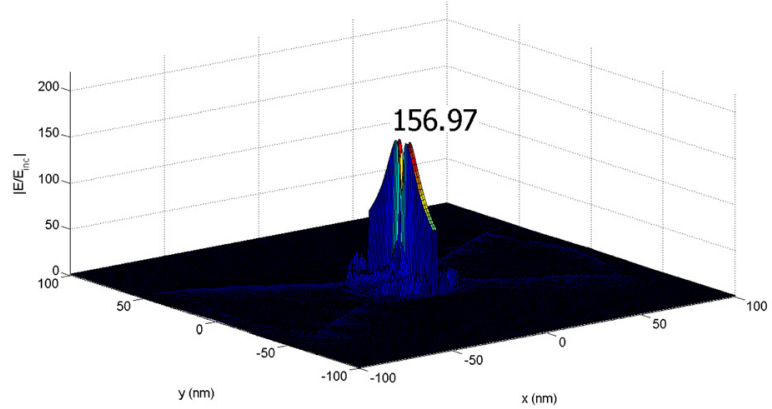

(a) $r=90 \mathrm{~nm}$

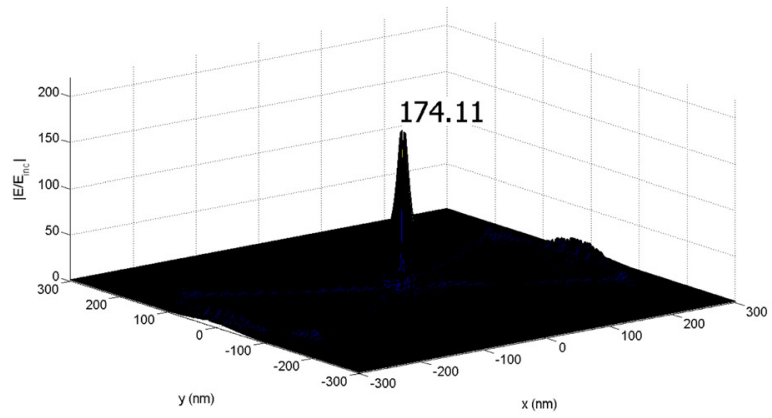

(c) $r=282.3 \mathrm{~nm}$

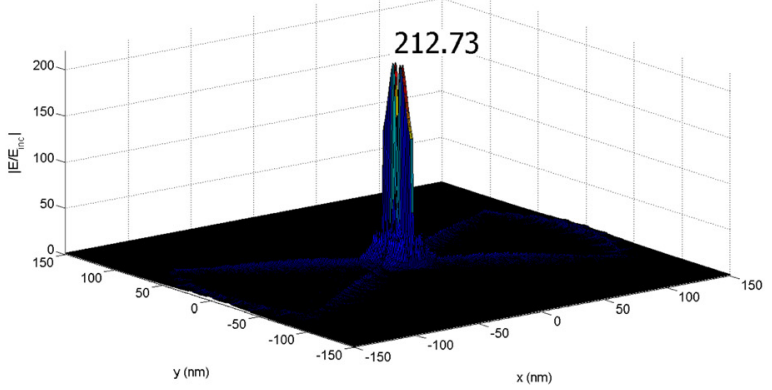

(b) $r=141.2 \mathrm{~nm}$

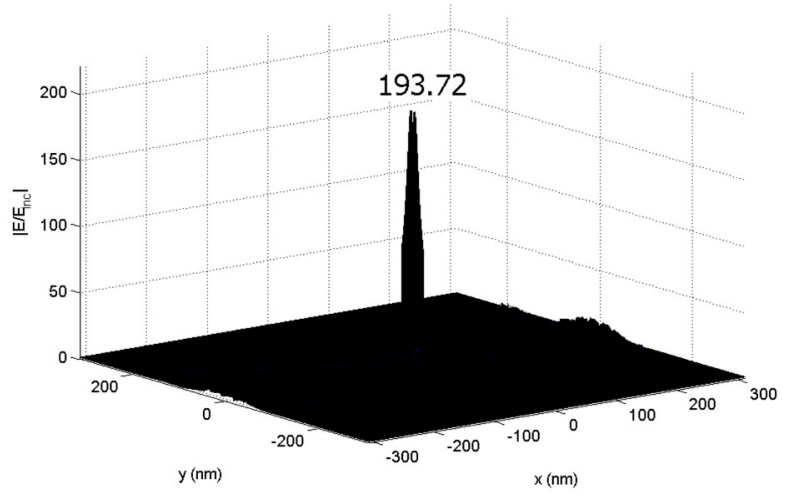

(d) $r=300 \mathrm{~nm}$

FIG. 5. (Color online) The E-field enhancement factors on the surfaces $1 \mathrm{~nm}$ above the surfaces of the bowtie-shaped dimers of different radii, $g=3 \mathrm{~nm}$. The wavelength of the surface plasmonic waves is $564.6 \mathrm{~nm}$, which is calculated based on Eqs. (1) and (2).

the optimal $r$ is the same as that of the one aligned to the $x$-axis. When all the dimers of radius $r$ are superposed at different $\phi$ 's, as shown in Fig. 1, they all have excited surface plasmons and resonate with the structure, and the E-field enhancement becomes stronger. As an example, in Fig. 1, the central angle is set to be $60^{\circ}$ and three dimers are superposed, forming a pancake-shaped aggregate.

\section{NUMERICAL RESULTS}

Poggio-Miller-Chang-Harrington-Wu-Tsai (PMCHWT) method is used to predict and analyze the performances of the proposed structure, which is a penetrable structure at optical frequencies. This approach provides high accuracy in the near field calculation with structures that have sharp edges or

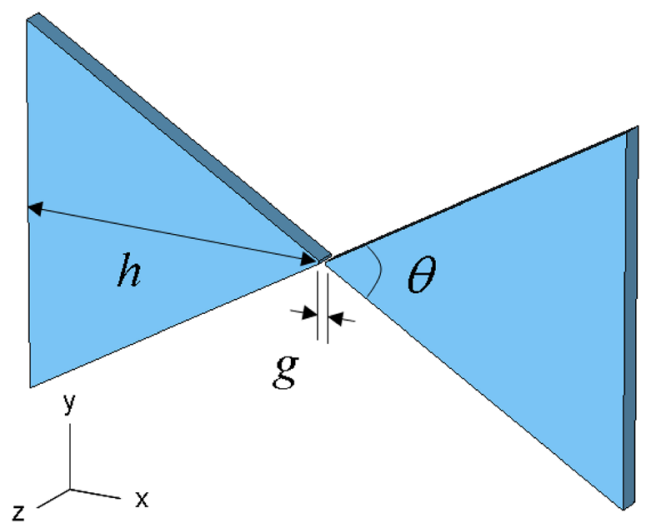

FIG. 6. (Color online) A dimer with triangles at the cross section. tips, due to the nature of this method. The details of the calculation method are included in the supporting materials. ${ }^{19}$

In the simulations, a plane wave at $500 \mathrm{THz}$ illuminates the object, $\mathbf{k}_{i n c}=-k_{z} \hat{z}, \mathbf{E}_{i n c}=E_{x} \hat{x}$. Gold is used as the material for the nanoparticles. Its dielectric constant is $-8.59+$ $1.3 i{ }^{18}$ Therefore, based on Eq. (2), the radius of the sector of the dimer $r$ is determined to be $141.2 \mathrm{~nm}$.

Figure 5 shows the E-field enhancement factors (normalized E-fields) on the surfaces $1 \mathrm{~nm}$ above the surfaces of

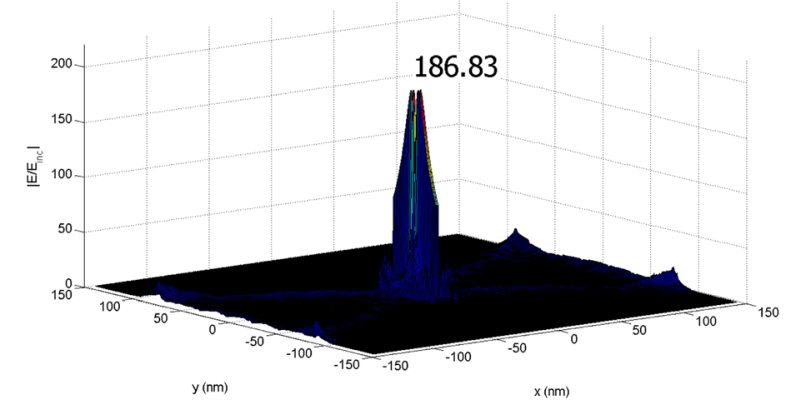

FIG. 7. (Color online) The E-field enhancement factors on the surfaces $1 \mathrm{~nm}$ above the surfaces of the dimer in Fig. $6(\mathrm{~g}=3 \mathrm{~nm})$.

TABLE I. Performances of the bowtie-shaped dimers.

\begin{tabular}{lccc}
\hline \hline$r(\mathrm{~nm})$ & $d(\mathrm{~nm})$ & $t(\mathrm{~nm})$ & $|\mathbf{E}|_{\max } /|\mathbf{E}|_{\text {inc }}$ \\
\hline 141.2 & 1 & 22.9 & 489.68 \\
141.2 & 3 & 22.9 & 212.73 \\
141.2 & 6 & 22.9 & 105.27 \\
\hline
\end{tabular}




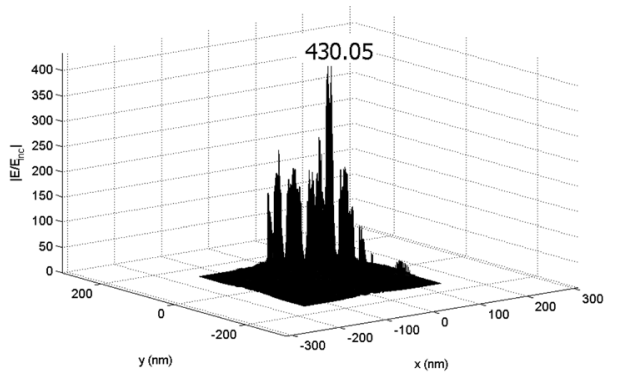

(a) $3 \mathrm{D}$ view

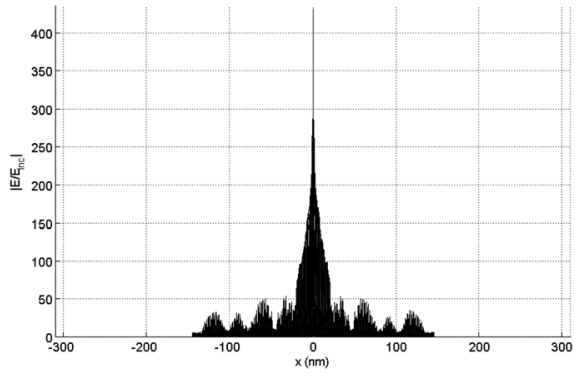

(b) $2 \mathrm{D}$ view
FIG. 8. The E-field enhancement factors on the surfaces $1 \mathrm{~nm}$ above the surfaces of the proposed aggregate in Fig. 1. the bowtie-shaped dimers of different radii. The size of the gap is fixed to be $3 \mathrm{~nm}$. As shown in Fig., when the radius equals to $141.2 \mathrm{~nm}$, a quarter of the guided wavelength of the surface plasmonic waves in the particle, maximum E-field enhancement can be obtained at 212.73. The maximum E-fields on these surfaces are also the maximum E-fields in all, based on the simulated results.

A dimer with two triangles at the cross section, as shown in Fig. 6, is calculated for the purpose of comparison. The height $h$ is set to be $141.2 \mathrm{~nm}$, where structure resonance can be expected in the $\mathrm{x}$-direction and $g=3 \mathrm{~nm}$. Figure 7 shows the E-field enhancement factor of this structure on the same surface ( $1 \mathrm{~nm}$ above the surface of the dimer). As can be seen in Fig. 7, this triangular dimer shows a maximum E-field enhancement of 186.83. It is less than the bowtieshaped structure with a radius of $141.2 \mathrm{~nm}$ and the same gap size (Fig. 5(b)). This is because, in the triangular dimer, the dimension of the dimer is not half of the guided wavelength in the directions other than the $x$-direction and there is a lack of resonance in these directions.

In the literature, it is reported that the enhancement factor of a dimer is inversely proportional to the gap size. ${ }^{3}$ Table I shows the maximum E-field enhancement factors of the dimers with different gap sizes. When the gap size goes as small as $1 \mathrm{~nm}$, the E-field can go as high as 489.68. It can be seen that a smaller gap size enhances coupling between the two particles and greatly strengthens the E-field enhancement. However, in the real world, a small gap size is challenging for fabrication.

Figure 8 shows the E-field enhancement factors on the surface $1 \mathrm{~nm}$ above the surface of the proposed aggregate $(r=141.2 \mathrm{~nm}, g=3 \mathrm{~nm})$, including a $3 \mathrm{D}$ and a $2 \mathrm{D}$ view. In the figure, this aggregate exhibits an enhancement factor of more than 430.05. Clearly, it shows the significant effect of the superposition on the enhancement of E-fields. When three dimers are superposed into an aggregate, the enhancement is greatly strengthened and the enhancement factor is more than being doubled. The asymmetry of fields along the $y$-direction is observed, and the maximum point is shifted from the point where $y=0$. They are probably due to the waveguiding effect formed by two particles that are sitting next to each other. Comparing the dimer with a $1 \mathrm{~nm}$ gap and the proposed aggregate, we see that, with the proposed structure, high enhancement can be obtained without pushing two particles to be very close.

\section{CONCLUSION}

In this letter, we proposed a pancake-shaped aggregate that effectively focuses surface plasmons. It is a superposition of bowtie-shaped dimers, in which surface plasmons are excited, structurally resonated, and coupled. The performance of the proposed structure is predicted using the integral equation method, which can accurately calculate the nearfields of nanoparticles. The proposed structure is predicted to have an E-field enhancement of more than 400 times. The proposed structure offers an approach to obtain a high enhancement factor without pushing two particles unpractically close in a dimer. It is a promising structure that can be applied for single molecule detections by using surfaceenhanced Raman scattering. It is useful in the area of nanooptics, where high E-fields are needed. There are many more possible designs by taking this proposed approach. For example, the central angle of each sector, $\theta$, the number of dimers for superposing can be further optimized for further improvement of the performance.

${ }^{1}$ S. Nie and S. R. Emory, Science 275, 1102 (1997).

${ }^{2}$ K. Kneipp, Y. Wang, H. Kneipp, L. T. Perelman, I. Itzkan, R. R. Dasari, and M. S. Feld, Phys. Rev. Lett. 78, 1667 (1997).

${ }^{3}$ E. Hao and G. C. Schatz, J. Chem. Phys. 120, 357 (2004).

${ }^{4}$ K. Li, L. Clime, B. Cai, and T. Veres, Nanotechnology 19, 145305 (2008).

${ }^{5}$ R. Jin, Y. Cao, C. A. Mirkin, K. L. Kelly, G. C. Schatz, and J. G. Zhang, Science 294, 1901 (2001).

${ }^{6}$ C. L. Haynes and R.P. Van Duyne, J. Phys. Chem. B 105, 5599 (2001).

${ }^{7}$ S. Zou and G. C. Schatz, Chem. Phys. Lett. 403, 62 (2005).

${ }^{8} \mathrm{~K}$. Kneipp, M. Moskovits, and H. Kneipp, Surface-Enhanced Raman scattering, Physics and Applications (Springer, New York, 2006), Chap. 1.

${ }^{9}$ N. Yu, E. Cubukcu, L. Diehl, D. Bour, S. Corzine, J. Zhu, G. Hofler, K. B. Crozier, and F. Capasso, Opt. Express 15, 13272 (2007).

${ }^{10}$ J. Li, D. Fattal, and Z. Li, Appl. Phys. Lett. 94, 263114 (2009).

${ }^{11} \mathrm{~K}$. Li, L. Clime, L. Tay, B. Cai, M. Geissler, and T. Veres, Anal. Chem. 80, 4945 (2008)

${ }^{12}$ K. Li, M. I. Stockman, and D. J. Bergman, Phys. Rev. Lett. 91, 227402 (2003).

${ }^{13}$ M. D. Malinsky, K. L. Kelly, G. C. Schatz, and R. P. V. Duyne, J. Phys. Chem. B 105, 2343 (2001).

${ }^{14}$ S. Y. Huang, B.-I. Wu, B. Zhang, Y. H. Lee, V. Liberman, and M. Rothschild, J. Appl. Phys. 106, 114306 (2009).

${ }^{15}$ J. A. Kong, Electromagnetic Wave Theory, (EMW, Cambridge, MA, 2008).

${ }^{16} \mathrm{C}$. A. Balanis, Antenna Theory Analysis and Design, 3rd ed. (Wiley, New York, 2005), pp. 32-33.

${ }^{17}$ H. Raether, Surface Plasmons on Smooth and Rough Surfaces and on Gratings, Springer Tracts in Modern Physics, Vol. 111 (Springer, New York, 1988).

${ }^{18}$ E. D. Palik, Handbook of Optical Constants of Solids (Academic, San Diego, 1998), Chap. 2.

${ }^{19}$ See supplementary material at http://dx.doi.org/10.1063/1.3681377 for details of the calculation method. 